\title{
Educação permanente através de um sistema m-Health voltado ao controle da obesidade em adultos
}

\author{
Maria Lúcia Kroeff Barbosa ${ }^{1}$, Sílvio César Cazella ${ }^{1,2}$, Márcia Rosa da Costa ${ }^{2}$, \\ Emilian Rejane Marcon ${ }^{3}$, Valter Roesler ${ }^{4}$ \\ ${ }^{1}$ Programa de Pós-Graduação em Informática na Educação - UFRGS \\ ${ }^{2}$ Programa de Pós-Graduação em Ensino na Saúde - UFCSPA \\ ${ }^{3}$ Hospital de Clínicas de Porto Alegre - HCPA \\ ${ }^{4}$ Instituto de Informática - UFRGS \\ malukroeff@yahoo.com.br, silvio.ufcspa@gmail.com, \\ marciarc.ufcspa@gmail.com, emarcon@hcpa.edu.br, roeslerdinf.ufrgs.br
}

\begin{abstract}
Resumo. Este artigo tem como objetivo apresentar um sistema de apoio à educação em saúde voltado ao tratamento e prevenção da obesidade em adultos, denominado Emagreça@Saudável. Três teorias principais embasam o sistema: a) o Modelo Transteórico de Mudança; b) as tecnologias persuasivas para mudança de comportamento visando a adoção de hábitos saudáveis; c) os princípios de design para dispositivos móveis. O sistema visa suprir funcionalidades e indicar mecanismos inovadores para apoio à reeducação alimentar e motivação da prática de atividades físicas, com a intenção de servir de apoio a ações de saúde pública na prevenção e controle da epidemia da obesidade.
\end{abstract}

Palavras-Chave: $m$-Health, obesidade, educação em saúde, aplicativo

\section{Permanent education through an m-Health system for the control of obesity in adults}

\begin{abstract}
This article aims to present a system of assistance to health education aimed at the treatment and prevention of obesity in adults, called Slim\&Healthy. Three main theories support the system: a) the Transtheoretical Model of Change; b) persuasive technologies for behavior change aiming at the adoption of healthy habits; $C$ ) the design principles for mobile devices. The system aims to provide functionalities and indicate innovative mechanisms to support dietary reeducation and motivation to practice physical activities, with the intention of supporting public health actions in the prevention and control of the obesity epidemic.
\end{abstract}

Keywords: $m$-Health, obesity, health education, app

\section{Introdução}

O uso das Tecnologias da Informação e Comunicação (TICs) para cuidados com a saúde mediante a utilização de dispositivos móveis é denominado de Mobile-Health ou $m$-Health [WHO, 2012], e sua utilização vem aumentando ao longo do tempo. Conforme Morris [2012], este fato pode ser explicado pela concentração de três fatores: o aumento das doenças crônicas, redução do acesso aos cuidados clínicos, e as 
inovações no âmbito das tecnologias móveis, que auxiliam no processo de aprendizagem informal e favorecem a autoaprendizagem, as relações sociais, $\mathrm{o}$ compartilhamento de experiências e a aprendizagem colaborativa, proporcionando o acesso ao conhecimento e oportunizando ao usuário ser um agente ativo e responsável pelo seu tratamento e cuidados relacionados à sua saúde [Costa et al., 2014]. Na tentativa de compreender e criar sistemas tecnológicos persuasivos que sejam efetivos, estudos na área de saúde e tecnologia estão surgindo.

Sendo assim, este artigo tem como objetivo apresentar o desenvolvimento de um sistema de apoio à educação em saúde voltado ao tratamento e prevenção da obesidade em adultos, intitulado Emagreça@Saudável. O texto encontra-se organizado em 5 seções, incluídas a Introdução e Conclusão. A seção 2 descreve estudos científicos que abordam o uso de aplicativos com foco na perda de peso. A seção 3 apresenta a fundamentação teórica, no contexto da saúde, na qual o sistema proposto está baseado, e a seção 4 traz a proposta de interfaces para o aplicativo (app) e considerações sobre a mesma.

\section{Aplicativos móveis para o tratamento e controle da obesidade}

Uma revisão sistemática da literatura, baseada nas diretrizes do Ministério da Saúde [2012], foi realizada com objetivo de identificar publicações científicas sobre aplicativos móveis para o controle e tratamento da obesidade. Foram utilizadas as seguintes expressões de busca: "mhealth application", "ehealth application", "adult overweight", "adult obesity" nas bases de dados disponibilizadas pelos portais PubMed Health, BVS (Biblioteca Virtual em Saúde) e Cochrane Library, no período de janeiro de 2013 a setembro de 2016. Foram incluídos artigos disponíveis na versão completa, do tipo ensaio clínico e realizados com adultos acima de 18 anos, e que descrevessem resultados relativos ao controle do excesso de peso (IMC $\geq 25 \mathrm{~kg} / \mathrm{m}^{2}$ ) ou obesidade (IMC $\geq 30 \mathrm{~kg} / \mathrm{m}^{2}$ ) por meio de variáveis como medidas de peso, IMC ou circunferência abdominal. Mulheres grávidas, adultos com incapacidade de realizar atividade física ou doença mental grave, artigos sem resultado de implementação ou duplicados foram excluídos. Como resultados, obtiveram-se os artigos descritos a seguir.

O TXT2BFiT [Hebden et al., 2013] foi um dos primeiros programas $m$-Health projetados para jovens adultos (18-35 anos) com eficácia demonstrada no controle do peso. Utiliza componentes como ligações de treinamento personalizado que visam estabelecer metas personalizadas com os participantes, a fim de eliminar as barreiras pessoais para a mudança de comportamento e manter a motivação dos participantes para usar os materiais adicionais disponibilizados pelo programa, tais como aplicativos de auto monitoramento e acesso ao site. Além disso envia mensagens de texto e emails. Para comprovar os benefícios desse programa, outro estudo foi realizado [Partidge et al., 2016], e o grupo intervenção relatou uso elevado de chamadas de treinamento, mensagens de texto e e-mails. As ligações de treinamento foram relatadas como úteis, atuando como motivador externo. $\mathrm{O}$ engajamento no uso do site e aplicativos sugeridos foi considerado fraco. Como sugestão, foi solicitado incorporar os aplicativos de auto monitoramento e recursos disponíveis no site em um único aplicativo de smartphone.

O aplicativo MMM (My Meal Mate) [Carter et al., 2013] utiliza uma abordagem comportamental baseada em evidências. Um estudo piloto randomizado priorizou os componentes face-a-face com os usuários no momento da inscrição, em 6 semanas e no 
término do estudo (6 meses), a fim de realizar medidas antropométricas e questionários. Os participantes foram distribuídos aleatoriamente para formar 3 grupos: grupo Smartphone recebeu um smartphone HTC Desire com o aplicativo MMM instalado; grupo Diário Alimentar recebeu um diário alimentar em papel, um livro de contagem de calorias e uma calculadora; grupo Website recebeu um voucher para 6 meses de acesso ao site da Weight Loss Resources. O estudo piloto mostrou que o app MMM pode ser uma intervenção viável a aceitável para auxiliar na perda de peso.

Martin et al. [2015] desenvolveram um app denominado SMARTLOSS ${ }^{\mathrm{SM} \text {, }}$ também baseado na teoria comportamental e que visa fornecer monitoramento de progresso remoto, além de recomendações de tratamento e materiais informativos através das capacidades multimídias dos smartphones. No estudo piloto randomizado controlado, com duração de 3 meses, o grupo que utilizou o app apresentou perda de peso maior do que o grupo controle. Os resultados obtidos no estudo sugerem que esse app e intervenções de perda de peso semelhantes podem fornecer métodos eficazes para auxiliar em tratamentos com o mesmo propósito.

Por fim, o Cell Phone Intervention for You (CITY) baseia-se na teoria social cognitiva e modelo transteórico [Svetkey et al., 2015]. O estudo com um grupo controle e dois grupos com níveis de interação diferentes concluiu que uma intervenção eficaz exige maior eficiência da tecnologia móvel, apoio social e interação humana, além de uma abordagem adaptativa ao design de intervenção. O uso do smartphone foi utilizado para auto monitoramento de peso, consumo alimentar e atividade física. Para um dos grupos interação, haviam sessões presenciais semanais com nutricionistas, contatos telefônicos mensais com fixação de metas, desafios e apoio social.

Pelos estudos apresentados, verifica-se a importância de combinar as diversas maneiras de utilizar o $m$-Health para um atendimento de saúde mais eficiente e eficaz.

\section{Teorias com foco na mudança de comportamento}

Existe uma grande variedade de apps desenvolvidos no contexto da saúde. Dados atualizados do Research2Guidance [2016] apontam que os com maior potencial de sucesso para o m-Health estão relacionados ao tratamento de doenças crônicas como diabetes e obesidade. Entretanto, comportamentos distintos exigem diferentes estratégias e técnicas. As tecnologias desenvolvidas intencionalmente para mudar os hábitos de uma pessoa envolvem diversos campos como a psicologia social, tecnologia da informação, design, entre outras áreas correlatas e são chamadas de tecnologias persuasivas [Fogg, 2011]. Uma das teorias que vem se mostrando mais promissora no que tange a entender e explicar a maioria dos comportamentos em saúde é a que aborda o Modelo Transteórico de Mudança de Comportamento [Prochaska \& DiClemente, 1982], criada por meio da análise comparativa de 29 teorias e modelos dos principais enfoques psicoterápicos, tais como teorias cognitivo-comportamental, humanista, psicanálise e Gestalt. A principal ideia é de que as automudanças bem-sucedidas dependem da aplicação de estratégias certas (processos) na hora certa (estágios).

Para a o desenvolvimento do sistema Emagreça@Saudável, além da teoria do Modelo Transteórico de Mudança, buscou-se embasamento teórico no Modelo de Comportamento de Fogg e nas diretrizes de Margaret Morris, com foco nas tecnologias persuasivas para mudança de comportamento visando a adoção de hábitos saudáveis. Em termos de interface com o usuário, levaram-se em consideração os princípios de 
design sugeridos por Inostroza et al. [2013], voltados para interfaces de smartphones sensíveis ao toque com foco na usabilidade. As próximas subseções detalham as ideias dos autores citados acima.

\subsection{Teoria do Modelo Transteórico de Mudança}

Prochaska, DiClemente \& Norcross [1992] salientam que o Modelo Transteórico de Mudança (MTT) visa compreender de que forma as pessoas realizam mudanças duradouras em suas vidas e que as mesmas passam por diferentes estágios até que se perceba as mudanças nas atitudes, intenções e comportamentos, além da motivação existente para que ocorram essas modificações. Enquanto o tempo que uma pessoa pode ficar em cada estágio é variável, as tarefas necessárias para passar para a próxima fase não são. Os estágios são especificados pelos autores em: pré-contemplação (indivíduo não tem a intenção de mudar o comportamento pelos próximos seis meses, pelo menos); contemplação (indivíduo consciente que existe um problema e pensa seriamente em superá-lo, mas não está ainda no compromisso de agir); preparação (indivíduo pretende agir no futuro imediato. Normalmente, já tomou alguma ação significativa, mas ainda não atingiu uma ação efetiva); ação (indivíduo fez modificações específicas em seu estilo de vida; manutenção (indivíduo fez modificações específicas em seu estilo de vida e está trabalhando para impedir a recaída).

\subsection{Modelo de Comportamento de Fogg}

O modelo de comportamento de Fogg [2011], ou Fogg's Behavior Model (FBM), além de explicar como ocorre o processamento cognitivo, visa indicar como se pode mudar determinados comportamentos usando tecnologias persuasivas. Segundo o autor, os principais fatores que afetam por qual rota (periférica ou central) um indivíduo usa para processar uma mensagem são a motivação (desejo de processar a mensagem), a habilidade (capacidade para avaliação crítica), e o gatilho (um estímulo, externo ou interno, que sinalize a ação e indica o momento ideal para realizar determinada ação). Outros pontos importantes para a criação de uma tecnologia persuasiva que seja efetiva levam em conta seu propósito e a relevância da simplicidade dentro da mesma, a fim de tornar a experiência mais intuitiva.

\subsection{Sete Diretrizes de Morris}

Margaret Morris [2012] propõe sete diretrizes baseadas em uma série de técnicas que foram avaliadas tanto para a intervenção em saúde, como para a comunicação persuasiva, com foco na adoção de hábitos saudáveis por intermédio dos aplicativos móveis:

1) Fazer as pessoas lembrarem quem elas querem ser: esclarece duas maneiras eficazes de recorrer aos ideais pessoais (contraste entre valores/comportamento e reforço positivo).

2) Promover uma aliança: os dispositivos devem conhecer os objetivos do seu usuário e apontar a direção correta.

3) Aplicar influência social: narrativas detalhadas ou vídeos que retratam as estratégias bem-sucedidas de outras pessoas, no mesmo contexto ou parecido, podem influenciar o comportamento e promover a auto eficácia. 
4) Mostrar as pessoas aquilo que elas podem perder: as pessoas precisam de ajuda para compreender as perdas de saúde que poderiam sofrer como resultado de autocuidados pobres.

5) Colocar a mensagem onde a ação está: importância da mensagem no contexto de uso, ou seja, quanto mais próximo do tempo e local da atividade alvo que um lembrete ocorre, mais eficaz ele será.

6) Aumentar a consciência emocional: computação em nuvem facilita a análise de dados individuais e de grupo, permitindo um rápido feedback aos indivíduos sobre suas próprias tendências e como eles se comparam aos outros, possibilitando uma interferência na motivação dos mesmos

7) Reformular desafios: examinar a reação das pessoas aos eventos diários e considerar alternativas, ajuda-as a responder situações desafiadoras de maneira mais construtiva.

Considera-se que, para o desenvolvimento do Emagreça@Saudável, essas diretrizes podem auxiliar no entendimento das maneiras pelas quais os dispositivos móveis podem interagir com o usuário, a fim de incentivar mudanças comportamentais relacionadas à saúde.

\subsection{Princípios de Design}

Em termos de interface com o usuário, os princípios de design sugeridos por Inostroza et al. [2013], voltados para interfaces de smartphones sensíveis ao toque com foco na usabilidade, foram os considerados na construção do aplicativo. Tais princípios estão baseados nas heurísticas de Nielsen e a metodologia para sua definição apoiou um processo de desenvolvimento e refinamento confiável, totalizando um conjunto de 12 heurísticas que serão melhor abordadas na próxima seção.

\section{Emagreça@Saudável}

O Emagreça@Saudável trata-se de um sistema de apoio à educação em saúde com foco no tratamento da obesidade de adultos. É resultado de uma parceria que conta com uma rede formada por profissionais de diversas áreas, entre elas: educação física, informática, informática na educação, epidemiologia, psicologia e educação em saúde. A Figura 1 ilustra o sistema Emagreça@Saudável.

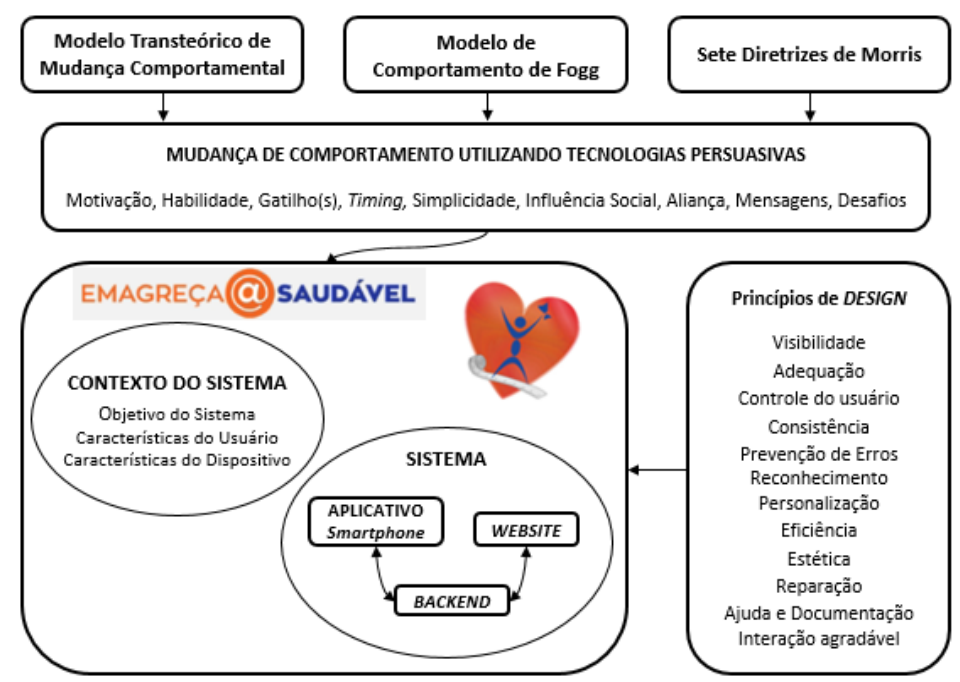

Figura 1: Sistema Emagreça@Saudável 
As teorias e princípios especificados na seção 3 foram analisados em conjunto com o Contexto do Sistema, que trata da importância de conhecer os fatores internos e externos que podem influenciar a experiência de uso. Nesse ínterim, consideram-se:

- Objetivo do Sistema: disponibilizar um aplicativo para dispositivos móveis que envolva cuidados com a saúde, com incentivos a mudanças individuais e de comportamento para prevenção e tratamento da obesidade, apoiando ações de promoção e educação em saúde.

- Características do Usuário: informações gerais das pessoas que vão utilizar (idade, gênero, grau de escolaridade, etc.), bem como suas necessidades (estado de saúde), intenções e meio o qual se encontram.

- Características do Dispositivo: qualidades e limitações de uso de um smartphone.

Já a composição do Sistema se faz pelos seguintes elementos:

a) Aplicativo para dispositivos móveis: interface principal, por onde o usuário cadastra seus dados, executa os desafios propostos, acompanha sua evolução e recebe orientações diariamente. Os dados do mesmo são armazenados localmente, e também enviados ao backend para gerar as estatísticas.

b) Website: servirá de apoio aos usuários do aplicativo, com repositório de vídeos, áudios e texto com dicas de saúde, esclarecimentos sobre estilo de vida saudável, bem como sugestões de aulas diferenciadas conforme perfil do usuário. No website, o usuário também poderá visualizar sua evolução, pois o mesmo também se comunica com o backend.

c) Área administrativa do Website: o website terá uma área administrativa que se comunica com o backend, buscando informações dos vários usuários e gerando estatísticas. Será possível agrupar usuários em grupos de acordo com seus perfis, e enviar mensagens ao dispositivo móvel de todos no grupo. As mensagens serão enviadas através do backend.

d) Um Backend: possui o banco de dados que armazena todas as informações dos usuários, servindo como apoio para a comunicação com os dispositivos móveis e também com o website.

A Tabela 1 detalha cada um dos principais elementos levados em consideração para as Tecnologias Persuasivas.

Tabela 1. Elementos priorizados para Persuasão

\begin{tabular}{|l|l|}
\hline \multicolumn{2}{|c|}{ Persuasão } \\
\hline \hline Gatilho (Trigger) & Deve ser claro e estar associado a determinada ação, ocorrendo em momento oportuno. \\
\hline Timing & $\begin{array}{l}\text { Momento certo para a ação. Deve estar de acordo com a motivação e a capacidade do usuário de realizar } \\
\text { a ação sugerida. Sem ele, o gatilho não tem efeito. }\end{array}$ \\
\hline Simplicidade & $\begin{array}{l}\text { Funções e exigências do aplicativo para realizar determinadas tarefas não devem ser vistos como } \\
\text { barreiras. }\end{array}$ \\
\hline Influência Social & $\begin{array}{l}\text { Integração por meio do apoio aos usuários, que pode vir do próprio sistema, ou quando esse permite a } \\
\text { influência de terceiros }\end{array}$ \\
\hline Aliança & Reconhecimento dos objetivos do usuário e apontar a direção correta. \\
\hline Mensagens & Colocar as mensagens no contexto da ação. \\
\hline Reformular desafios & Examinar a reação das pessoas aos eventos diários e considerar alternativas. \\
\hline
\end{tabular}


Os Princípios de Design considerados estão explicados na Tabela 2.

Tabela 2. Elementos priorizados para o Design

\begin{tabular}{|l|l|}
\hline \multicolumn{2}{|c|}{ Design } \\
\hline \hline Visibilidade do sistema & Indica ao usuário sua localização e ações dentro do aplicativo. \\
\hline $\begin{array}{l}\text { Adequação com o } \\
\text { mundo real }\end{array}$ & $\begin{array}{l}\text { O sistema deve falar a linguagem do usuário e seguir as convenções do mundo real, exibindo as } \\
\text { informações de maneira lógica e natural. }\end{array}$ \\
\hline Controle do usuário & Usuário pode reverter uma ação, ou utilizar mais de um caminho para chegar onde deseja. \\
\hline Consistência & Usuário deve realizar as ações de forma familiar e sem muito esforço. \\
\hline Prevenção de Erros & Funcionalidades não disponíveis devem permanecer escondidas ou desativadas. \\
\hline Reconhecimento & Objetos, ações e opções que devem estar claras e visíveis. \\
\hline Personalização & Opções de configurações básicas e avançadas de acordo com as necessidades contextuais. \\
\hline $\begin{array}{l}\text { Eficiência de utilização } \\
\text { e desempenho }\end{array}$ & $\begin{array}{l}\text { Exibição das informaçoses necessárias em um tempo razoável. Animações e transições devem ser } \\
\text { exibidas sem problemas. }\end{array}$ \\
\hline Estética & $\begin{array}{l}\text { Tornar a experiência do usuário agradável. Deve- se evitar a exibição de informações indesejadas em } \\
\text { um contexto definido de uso. }\end{array}$ \\
\hline Reparação & $\begin{array}{l}\text { Exibição de mensagens de erro em um idioma familiar, indicando o problema de forma precisa e } \\
\text { sugerindo uma solução construtiva }\end{array}$ \\
\hline Ajuda & Documentação e ajuda fáceis de encontrar, centradas na tarefa atual e indicando etapas a seguir. \\
\hline Interação & $\begin{array}{l}\text { Botões físicos ou similares para as principais funcionalidades, localizadas em posições reconhecíveis e } \\
\text { que devem caber na postura natural das mãos do usuário. }\end{array}$ \\
\hline
\end{tabular}

A seguir, o detalhamento da construção das principais interfaces do aplicativo.

\section{Interfaces do Aplicativo}

Na primeira vez que o usuário entra no aplicativo, aparece uma mensagem de boasvindas e a necessidade de fazer o login, como mostra a Figura 2(a)(b). Após, informações de perfil, tais como idade, escolaridade, renda (informação opcional), nível de stress, se usa bebida/cigarro, grau de sedentarismo e medicamentos são solicitadas.

Além disso, medidas de peso, altura, cintura e quadril também são necessárias, uma vez que esses dados calculam o IMC (Índice de Massa Corporal) e o ICQ (Índice Cintura Quadril), que são importantes métricas quantitativas de acompanhamento da evolução do usuário em relação ao seu estado de saúde. A Figura 2 (c)(d) apresenta as telas iniciais para cadastro de conta via app, onde o usuário criará o seu perfil e poderá escolher como quer ser chamado, bem como sua aparência, seja por uma foto, uma imagem que poderá ser buscada nos arquivos ou até mesmo um avatar.

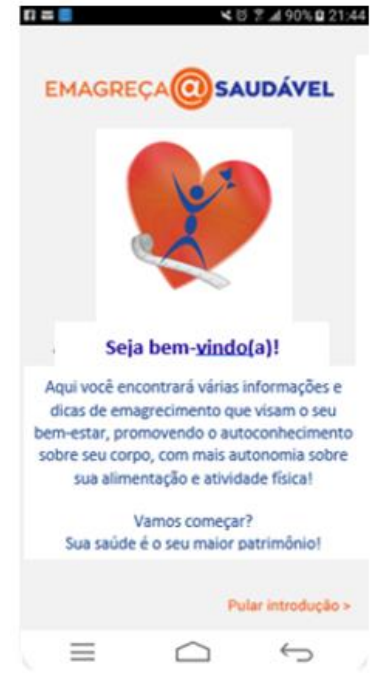

(a)

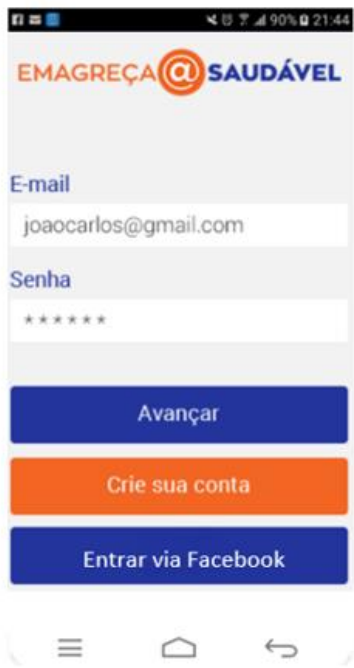

(b)

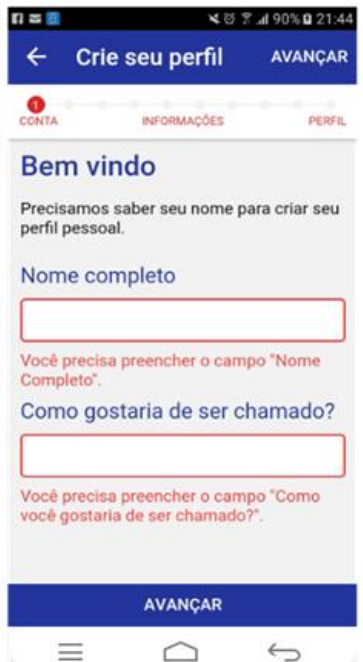

(c)

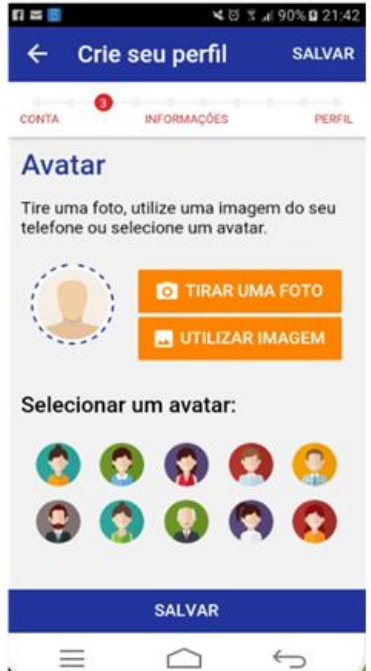

(d)

Figura 2: (a) Interface de boas-vindas; (b) Interface de entrada para o app; (c) Interface para cadastro via app; (d) Interface para criação do "Avatar" 
Como observa-se na Figura 2, estão presentes algumas das heurísticas de design, entre elas visibilidade do sistema, adequação com o mundo real, controle do usuário, personalização, reconhecimento, estética e reparação de erros. Por exemplo, é permitido ao usuário ver qual estágio de preenchimento das informações ele se encontra, o que pode ser visualizado na segunda barra superior, pelos círculos vermelhos. Também é permitido a voltar à tela anterior pela seta localizada na barra superior à esquerda da tela do dispositivo.

A tela de acompanhamento principal (dia-a-dia do usuário) é vista na Figura 3. A "Agenda diária" (Figura 3a) traz em destaque a meta a ser cumprida no dia, além de um desafio proposto para o mesmo dia. Tal interface também traz algumas derivações:

- Medições: monitoramento dos sinais vitais e medidas do participante para fins de definição de metas, acompanhamento e orientação (seja por uma equipe ou de forma automática).

- Alimentação: diferenciando-se dos outros aplicativos, a intenção não é que o usuário tenha que cadastrar toda refeição ingerida e ficar controlando calorias por meio de índices sem fim. A prioridade é trazer comparações e dicas de substituições de alimentos não saudáveis por mais saudáveis, bem como algumas sugestões e receitas, por exemplo, de sucos detox ou com alimentos funcionais.

- Atividade Física: nível de esforço físico sendo dispendido diariamente. Opção de cadastro para atividades físicas, bem como um contador de passos incluso no app que marca a quantidade de passos.

- Status e evolução: objetivo motivacional numa espécie de "gamificação", buscando comparar a evolução com outras pessoas com perfil similar.

- Mensagens: dependendo da evolução, grau de interação do participante, entre outros, o sistema envia mensagens automáticas.

- Social: integra o participante em redes sociais que permite ao mesmo trocar informações e mensagens com outras pessoas em situação similar, ou com interesse na troca de conhecimentos.

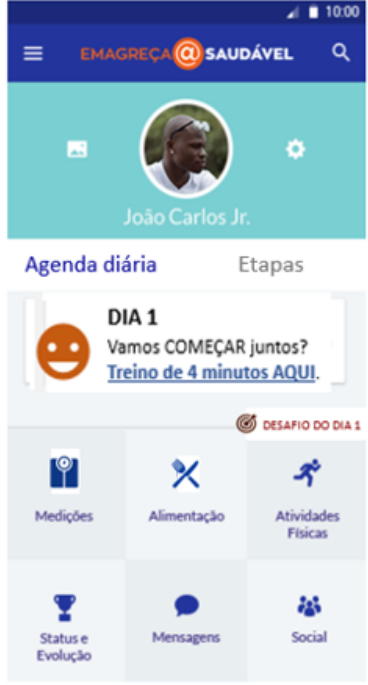

(a)

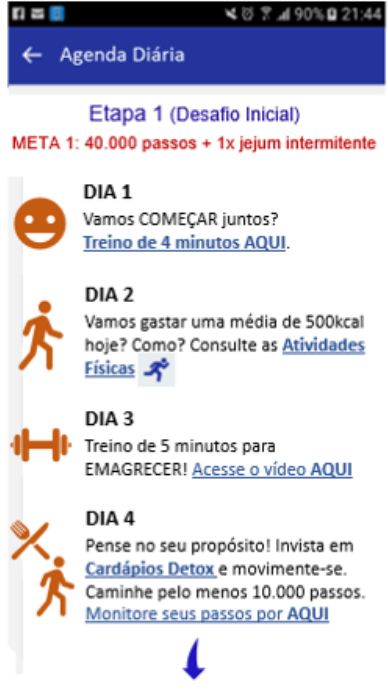

(b)

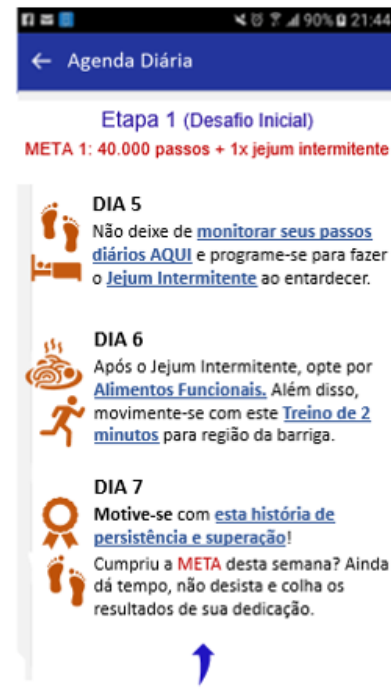

(c)

Figura 3: (a) Interface principal, com modelo de "Agenda diária"; (b)(c) Interface com modelo de Etapa 1 (Desafio Inicial) 
$\mathrm{Na}$ Figura 3(b)(c), a "Etapa 1" apresenta alguns dos objetivos a serem cumpridos, e será composta por 4 metas, pois a intenção é fazer com que o participante se comprometa a realizar as mesmas dentro de um período máximo de tempo e cumpra cada uma das etapas satisfatoriamente. Por exemplo, caminhar 40.000 passos e fazer o jejum intermitente, que significa ficar de 12 a $24 \mathrm{~h}$ sem se alimentar, contando o período do sono.

Para quase todas as derivações apresentadas nessa interface (exceto "Status e Evolução"), o aplicativo trará em forma de texto, áudios ou vídeos, informações e dicas que possam auxiliar os usuários a compreenderem as perdas de saúde que podem sofrer ao logo do tempo, caso optem por seguirem em uma rotina de autocuidados não satisfatória. Além disso, narrativas de estratégias bem-sucedidas de outras pessoas, no mesmo contexto ou parecido, também serão disponibilizadas em momentos oportunos.

\section{Conclusão}

O m-Health está em grande expansão e os aplicativos para saúde possuem grande potencial. Porém, mudar e manter novos hábitos não é tão simples. Com as ferramentas necessárias para promoção da saúde e bem-estar, o Emagreça@Saudável está embasado em teorias, modelos e diretrizes que possuem caráter persuasivo e pretende auxiliar seus usuários não só na mudança, mas na permanência de um comportamento saudável. $\mathrm{O}$ texto apresentado mostrou o estágio de desenvolvimento do sistema, com foco no aplicativo que está prestes a ser finalizado. A intenção é que o mesmo sirva de apoio a ações de saúde pública que visam prevenir e controlar a epidemia da obesidade, contemplando também uma parcela da população que não possui condições sociais e financeiras para aquisição de informações mais direcionadas e personalizadas ao seu perfil, além de acompanhamento remoto de profissionais especialistas a custo baixo.

A continuidade dessa pesquisa envolve uma avaliação da usabilidade da interface do aplicativo para posterior validação do sistema com pacientes oriundos de uma unidade básica de saúde, todos adultos e com diagnóstico de sobrepeso ou obesidade. A intervenção tem o propósito de analisar se as estratégias propostas para mudanças de comportamento, com foco na perda de peso e via o sistema tecnológico proposto, serão bem-sucedidas.

\section{Referências}

CARTER, M. C. et al. "Adherence to a smartphone application for weight loss compared to website and paper diary: pilot randomized controlled trial". Disponível em: $<$ https://www.ncbi.nlm.nih.gov/pmc/articles/PMC3636323/>. Acesso em agosto de 2016.

COSTA, A. A. L. et al. Recomendação Personalizada de Conteúdo para Suporte à Aprendizagem Informal no Contexto da Saúde. "RENOTE". V. 12 No 1, julho, 2014. Disponível em: <http://seer.ufrgs.br/index.php/renote/article/view/49840/31197>. Acesso em abril de 2017.

FOGG, B. J. "BJ Fogg's Behavior Model”, 2011. Disponível em: $<$ http://www.behaviormodel.org/>. Acesso em fevereiro de 2016.

HEBDEN, L. et al. "TXT2BFiT' a mobile phone-based healthy lifestyle program for preventing unhealthy weight gain in young adults: study protocol for a randomized 
controlled trial"'. 2013. Disponível em:

$<$ https://www.ncbi.nlm.nih.gov/pubmed/23506013>. Acesso em setembro de 2016.

INOSTROZA, R. et al. "Usability heuristics for touchscreen-based mobile devices: Update". Disponível em: <http://jcc2013.inf.uct.cl/wpcontent/proceedings/ChileCHI/Usability\%20Heuristics\%20for\%20Touchscreenbased\%20Mobile\%20Devices\%20Update.pdf>. Acesso em janeiro de 2017.

MARTIN, C. K. et al. "Efficacy of SmartLossSM, a smartphone-based weight loss intervention: Results from a randomized controlled trial". 2015. Disponível em: $<$ http://onlinelibrary.wiley.com/doi/10.1002/oby.21063/full $>$. Acesso em setembro de 2016.

MINISTÉRIO DA SAÚDE. "DIRETRIZES METODOLÓGICAS: elaboração de revisão sistemática e metanálise de ensaios clínicos randomizados". Brasília, DF: Editora do Ministério da Saúde, 2012. Disponível em:

$<$ http://bvsms.saude.gov.br/bvs/publicacoes/diretrizes_metodologicas_elaboracao_sis tematica.pdf $>$. Acesso em janeiro de 2016.

MORRIS, M. "Motivating change with mobile: seven guidelines", v.19, n. 3, p. 26-31. 2012. Disponível em: <http://pt.scribd.com/doc/92691136/Motivating-Change-WithMobile-Seven-Guidelines-2012>. Acesso em fevereiro de 2016.

PARTRIDGE, S. et al. "Process evaluation of TXT2BFiT: a multi-component mHealth randomised controlled trial to prevent weight gain in young adults". Disponível em: $<$ https://ijbnpa.biomedcentral.com/articles/10.1186/s12966-016-0329-2>. Acesso em setembro de 2016.

PROCHASKA, J. O.; DiCLEMENTE, C. Transtheorical therapy: Toward a more integrative model of change. "Psycotherapy: Theory, Research and Practice", v. 19, p. 276-288, 1982. Disponível em:

<http://www.hbftpartnership.com/documents/uploadResources/TranstheoreticalTProchaska1982.pdf $>$. Acesso em janeiro de 2017.

RESEARCH2GUIDANCE. "m-Health App Developer Economics 2016: The current status and trends of the m-Health app market", outubro, 2016. Disponível em: $<\mathrm{http}$ //research2guidance.com/r2g/r2g-mHealth-App-Developer-Economics2016.pdf $>$. Acesso em janeiro de 2017

SVETKEY, L. P. et al. Cell phone intervention for you (CITY): A randomized, controlled trial of behavioral weight loss intervention for young adults using mobile technology. Disponível em:

$<$ http://onlinelibrary.wiley.com/doi/10.1002/oby.21226/full $>$. Acesso em setembro de 2016.

WHO (World Health Organization). “eHealth". Genebra: 2012. Disponível em: $<$ http://www.who.int/ehealth/en/>. Acesso em agosto de 2015. 